\title{
Resource Allocation Games in Interference Relay Channels Invited Paper
}

\author{
Elena Veronica Belmega, Brice Djeumou and Samson Lasaulce
}

\begin{abstract}
In this paper we study a distributed network comprising an interference channel in parallel with an interference relay channel. Therefore each source node can use two frequency bands and has to implement a certain power allocation policy. An example of application of such a model is the case where the performance of terminals operating in unlicensed bands would be enhanced by being allowed to exploit an additional frequency band in which a relay is available. In this network model, each user is selfish and wants to maximize its Shannon transmission rate. We analyze two cases. In the first case, the relaying node is assumed to implement an amplify-andforward (AF) protocol while in the second case it implements the decode-and-forward (DF) protocol introduced by Cover and El Gamal. For both cases we analyze the existence and uniqueness issues of the equilibrium of the aforementioned power allocation games. Several interesting and new results are provided. In particular: 1. The existence of a Nash equilibrium is shown to be always guaranteed in the case of the $\mathrm{AF}$ protocol; 2. The performance of a user or the network does not necessarily increase with the transmit power available at the relay; 3 . We show that there is naturally a game in interference relay channels (even if the power allocation policy is fixed) when the DF protocol is used; this game is induced by the decentralized choice of the cooperation degree between each source node and the relay node.
\end{abstract}

Index Terms-Game theory, information theory, cognitive radio, interference channel, open spectrum access, power allocation game, relay channel.

\section{INTRODUCTION}

Over the last two decades, spectrum congestion has become more and more a critical issue. This is one of the reasons why major actors in this arena like the Federal Communications Commission has released important reports providing a legal framework for deploying technologies like ultra wideband [1] or cognitive radio [2]. The latter technology has benefited from a more general consensus in part because its way of re-using the spectrum generates much less interference than ultra wideband systems. Cognitive terminals, based on spectrum sensing capabilities, are envisioned to be able to opportunistically and efficiently re-exploit the spectrum "left-overs" of other systems. In particular, more and more wireless devices operate in unlicensed bands, which gives to cognitive terminals a particular interest in locally exploiting the unused spectral resources, for instance, to increase their individual transmission rate or quality of communication. The technical issues addressed in this paper fall within this framework. More specifically,

The authors would like to thank the Network of Excellence Newcomm++, Work Package 6.

E. V. Belmega, B. Djeumou and S. Lasaulce are with LSS (joint lab of CNRS, Supélec, Univ. Paris-Sud 11), Supélec, Plateau du Moulon, 91192 Gif-sur-Yvette Cedex, France, \{belmega,djeumou,lasaulce\}@1ss.supelec.fr. our main motivation is to acquire a better understanding of a system where two cognitive transmitters, each of them communicating with its respective receiver, are offered the opportunity to use an additional frequency band on which a relaying node is available to further increase the performance of the system. In order to analyze such a distributed system we exploit both game theory, which offers the theoretical framework to study the interaction between several decision makers, and information theory, which allows us to characterize the performance limits of a communication system and therefore to study a network of terminals implementing good codes. Indeed, we assume that the two transmitters can choose freely their own resource allocation policy in order to selfishly maximize their transmission rates and, for this purpose, compete for the additional frequency band and thus for the available relay node. Several key questions arise: What is the influence of the relaying protocol on the game formulation? Is there a predictable state (equilibrium) at which this system will operate? Is it unique?

The system under investigation is modeled by a frequency non-selective (FNS) interference channel (IC) [3] in parallel with an FNS interference relay channel (IRC) [4], [5]. To the best authors' knowledge, the closest contributions to those presented in this paper are [4], [5], [6], [7], [8]. In [6] the authors consider the multiuser power control problem in a frequency-selective IC, which is modeled as a noncooperative game where the players want to maximize their individual transmission rate. In [7] the authors study the same channel but in their formulation each user selfishly minimize its transmit power under a minimal achievable rate constraint. In [4], [5] the authors introduce the IRC and focus on evaluating achievable rate regions for this channel when the links are assumed to be FNS and for the decodeand-forward (DF) protocol of [9]. Here, we study a more complex channel and also adopt a different point of view than [4], [5]. Indeed, the game-theoretic formulation of the problem is particularly relevant here since the users are not only interacting because of interference in each of the two sub-channels, but they are also assumed to be cognitive and therefore able to observe their environment and react to it accordingly. The system and game framework considered in this paper are very similar to [8]. Compared to the work [8] written by the authors, several new key results have been obtained. First, in [8] only the amplify-and-forward (AF) protocol is considered. Here, we have started to generalize our approach to the decode-and-forward protocol. In contrast to [8], we prove the existence of a Nash equilibrium (NE) without using the time-sharing argument, which concavifies 
the utilities but introduces a parameter that is not necessarily fixed (contrarily to what is assumed in [8]). Furthermore we prove the existence of an optimal amplification factor at the relay and conduct an exhaustive study to illustrate how the constraints on the transmit powers modifies the equilibrium uniqueness analysis. We also show that the DF protocol naturally introduces a game though the notion of cooperation degree.

This paper is structured as follows. In Sec. II we describe the system under investigation. Then we study the power allocation game for two different scenarios. First we assume that the relay implements amplify-and-forward, in Sec. III and investigate the existence of an equilibrium state for the corresponding game in Sec. III-A. The uniqueness of such a state is analyzed in detail for a particular case where the amplification gain is assumed to be fixed (Sec. III-B). In this case, we also propose a Stackelberg formulation where the relay is the leader of the game and can tune the amplification gain to optimize certain performance criteria. Second, in $\mathrm{Sec}$. IV we introduce the power allocation game in the case where the relay uses the DF protocol. In contrast with the AF protocol, the interaction between the two users can no longer be described only by the power allocation policies over the two channels but another degree of freedom has to be considered (the cooperation degrees between the sources and the relay) and thus the game becomes more complex. In this case we investigate the existence of the equilibrium for two particular cases: the case of fixed cooperation degrees between the sources and the relay (Sec. IV-B) and the case of fixed power allocation policies over the two channels (Sec. IV-C). Numerical results are also provided in Sec. V and we end by several concluding remarks and open issues (Sec. VI).

\section{SYSTEM MODEL}

As illustrated in Fig. 1, the system under investigation comprises two source nodes $\mathcal{S}_{1}, \mathcal{S}_{2}$, each of them transmitting its own message to its respective destination node $\mathcal{D}_{1}$ or $\mathcal{D}_{2}$ in a certain frequency band (the notation (a) will be used to refer to it), which is assumed to be unitary. Additionally there is one relaying node $\mathcal{R}$ that is available in an additional and non overlapping frequency band (denoted by (b)), also unitary. The signals transmitted by $\mathcal{S}_{1}$ and $\mathcal{S}_{2}$ in the bands (a) and (b), denoted by $X_{1}^{(a)}, X_{1}^{(b)}, X_{2}^{(a)}, X_{2}^{(b)}$, are assumed to be independent and subject to power constraints:

$$
\forall i \in\{1,2\}, \mathbb{E}\left|X_{i}^{(a)}\right|^{2}+\mathbb{E}\left|X_{i}^{(b)}\right|^{2} \leq P_{i} .
$$

Let us denote by $\theta_{i}$ the fraction of the power that is used for the transmission in the band (b), such that

$$
\mathbb{E}\left|X_{i}^{(b)}\right|^{2}=\theta_{i} P_{i}
$$

Under these assumptions, and denoting by $g_{i j}$ the channel gains between $\mathcal{S}_{i}, \mathcal{D}_{j}$ on band (a), the received baseband signals write:

$$
\left\{\begin{array}{l}
Y_{1}^{(a)}=g_{11} X_{1}^{(a)}+g_{21} X_{2}^{(a)}+Z_{1}^{(a)} \\
Y_{2}^{(a)}=g_{22} X_{2}^{(a)}+g_{12} X_{1}^{(a)}+Z_{2}^{(a)}
\end{array}\right.
$$
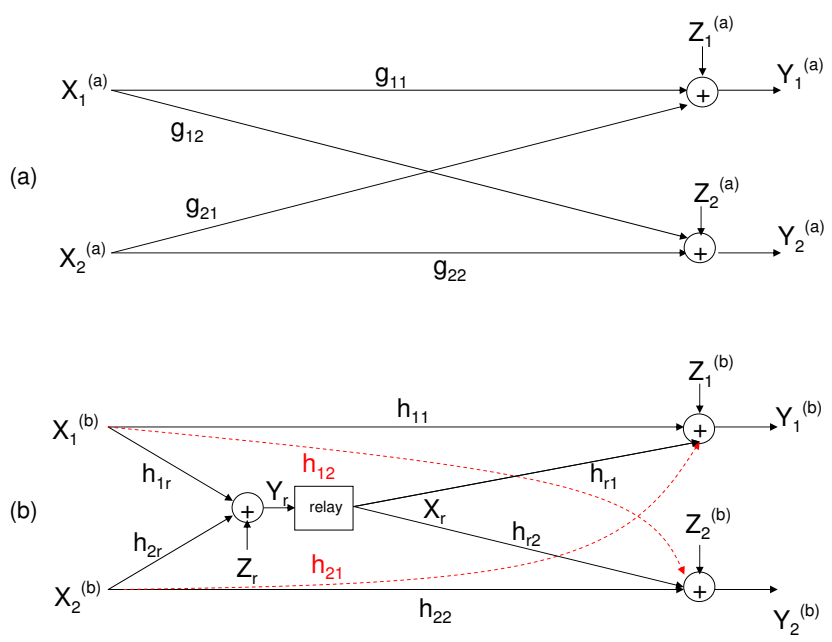

Fig. 1. System Model.

where $Z_{i}^{(a)}, i \in\{1,2\}$, are zero-mean additive white Gaussian complex noises of variance $N_{i}^{(a)}$. We assume that the system is distributed and that the two receivers treat the interference coming from the other users as additive noise. In this frequency band, the users achieve the following transmissions rates:

$$
\forall i, j \in\{1,2\}, R_{i}^{(a)}=C\left(\eta_{i}^{(a)}\right)
$$

where $C(x)=\log _{2}(1+x)$ is the capacity function and

$$
\eta_{i}^{(a)}=\frac{\left|g_{i i}\right|^{2} \rho_{i}^{(a)} \overline{\theta_{i}}}{\left|g_{j i}\right|^{2} \rho_{j}^{(a)} \frac{N_{j}^{(a)}}{N_{i}^{(a)}} \overline{\theta_{j}}+1},
$$

with $\rho_{i}^{(a)}=\frac{P_{i}}{N_{i}^{(a)}}$. We consider a realistic situation where only large scale propagation effects can be taken into account by the users to optimize their rates. Thus the channel gains are considered to be static. Concerning channel state information (CSI), we will always assume coherent communications between each source-destination pair $\left(\mathcal{S}_{i}, \mathcal{D}_{i}\right)$ whereas at the transmitters the information assumptions will be context-depending and therefore provided in the corresponding sections.

In the band (b), the received baseband signals and the achievable transmission rates, denoted by $R_{i}^{(b)}, i \in\{1,2\}$ depend on the relaying protocol used. In what follows we will focus on two different schemes: amplify-and-forward, where the relay simply amplifies its observation of the signals coming from the two sources and forwards it to the two destinations and decode-and-forward where the relay decodes both source messages.

\section{WHEN THE RELAY IMPLEMENTS THE AF PROTOCOL}

In this section we assume that the relay implements a zerodelay scalar amplify-and-forward (AF) protocol and operates 
in the full-duplex mode. Also we consider that the available transmission power at the relay is subject to the constraint $\mathbb{E}\left|X_{r}^{(b)}\right|^{2} \leq P_{r}$. Under these assumptions, and denoting by $h_{i j}$, for all $i, j \in\{1,2\}$, the channel gains between $\mathcal{S}_{i}, \mathcal{D}_{j}$ and $\mathcal{R}$ in band (b) respectively, the received baseband signals write:

$$
\left\{\begin{array}{l}
Y_{r}^{(b)}=h_{1 r} X_{1}^{(b)}+h_{2 r} X_{2}^{(b)}+Z_{r}^{(b)} \\
Y_{1}^{(b)}=a_{r} h_{r 1} Y_{r}^{(b)}+h_{11} X_{1}^{(b)}+h_{21} X_{2}^{(b)}+Z_{1}^{(b)} \\
Y_{2}^{(b)}=a_{r} h_{r 2} Y_{r}^{(b)}+h_{22} X_{2}^{(b)}+h_{12} X_{1}^{(b)}+Z_{2}^{(b)} .
\end{array}\right.
$$

The variances of the zero-mean additive white Gaussian complex noises $Z_{i}^{(a)}, Z_{i}^{(b)}, Z_{r}^{(b)}$ with $i \in\{1,2\}$ are denoted by $N_{i}^{(a)}, N_{i}^{(b)}, N_{r}^{(b)}$. At last $a_{r}$ corresponds to the relay amplification gain and is, in general, a function of $\theta_{1}$ and $\theta_{2}$. As the relay is subject to a power constraint the amplification gain $a_{r}\left(\theta_{1}, \theta_{2}\right)$ can not be chosen arbitrarily. By considering $a_{r}=\sqrt{\frac{P_{r}}{\mathbb{E}\left|Y_{r}^{(b)}\right|^{2}}}=\sqrt{\frac{P_{r}}{\left|h_{1 r}\right|^{2} P_{1} \theta_{1}+\left|h_{2 r}\right|^{2} P_{2} \theta_{2}+N_{r}^{(b)}}}$, one is ensured that the relay makes use of all its available power while meeting the power constraint; we will denote by $\bar{a}_{r}\left(\theta_{1}, \theta_{2}\right)$ the value of the amplification gain when this constraint is saturated. In Sec. III-B however, we will consider other values for this gain. We will focus on the situation where the gain is independent of $\theta_{1}$ and $\theta_{2}$. Two cases will be analyzed: the case where the constant amplification gain has to meet the power constraint, and the case where it is chosen to optimize a certain performance criterion (possibly with no power constraint).

Under these assumptions the transmission rate of user $i \in$ $\{1,2\}$ on channel (b) when the AF protocol is used by the relay is

$$
R_{i}^{(b)}=C\left(\eta_{i}^{(b)}\right)
$$

where

$$
\eta_{i}^{(b)}=\frac{\left|a_{r} h_{i r} h_{r i}+h_{i i}\right|^{2} \rho_{i}^{(b)} \theta_{i}}{\left|a_{r} h_{j r} h_{r i}+h_{j i}\right|^{2} \rho_{j}^{(b)} \frac{N_{j}^{(b)}}{N_{i}^{(b)}} \theta_{j}+a_{r}^{2}\left|h_{r i}\right|^{2} \frac{N_{r}^{(b)}}{N_{i}^{(b)}}+1}
$$

with $\forall i \in\{1,2\}, \rho_{i}^{(b)}=\frac{P_{i}}{N_{i}^{(b)}}$. Without loss of generality and for sake of clarity we will assume in the sequel that $\forall(i, s) \in$ $\{1,2\} \times\{a, b\}, N_{i}^{(s)}=N_{r}^{(b)}=N$. Also we introduce the quantities $\forall i \in\{1,2, r\}, \rho_{i}=\frac{P_{i}}{N}$.

\section{A. General power allocation game}

In this paper, one of our goals is to know how each transmitter is going to allocate its available power between the the IC and IRC, given the fact that they are able to observe each other and react accordingly. This situation of interaction corresponds to a game, and more precisely to a non-cooperative strategic-form game:

- the players of the game are the two transmitters;

- the strategy of transmitter $i$ consists in choosing $\theta_{i}$ in its strategy set $\mathcal{A}_{i}=[0,1]$ in order to maximize its individual rate;

- the utility function for user $i \in\{1,2\}$ is its achievable Shannon transmission rate given by $u_{i}\left(\theta_{i}, \theta_{-i}\right)=$
$R_{i}^{(a)}\left(\theta_{i}, \theta_{-i}\right)+R_{i}^{(b)}\left(\theta_{i}, \theta_{-i}\right)$, where $R_{i}^{(a)}$ and $R_{i}^{(b)}$ are the previously defined transmission rates.

Note that we implicitly assumed Gaussian codebooks for the two users since this choice is optimum for both or them. Also note that we will call state of the network the strategy profile of the users ${ }^{1}$. In this case the state of the system is the vector of power fractions that the users allocate to the IRC i.e., $\underline{\theta}=\left(\theta_{1}, \theta_{2}\right)$.

In distributed networks where users are selfish and free decision makers who interact with each other, a desirable feature is the existence of an equilibrium or a stable operating state of the system. In this respect, the Nash equilibrium (NE) [10] corresponds to a state of the network from which the users do not have any incentive to deviate unilaterally, because otherwise they would lose in terms of utility; this is translated mathematically by the following definition.

Definition 3.1: [Nash equilibrium] The state $\underline{\theta}^{*}$ is a pure $\mathrm{NE}$ if $\forall i \in\{1,2\}, \forall \theta_{i}^{\prime} \in \mathcal{A}_{i}, u_{i}\left(\theta_{i}^{*}, \theta_{-i}^{*}\right) \geq u_{i}\left(\theta_{i}^{\prime}, \theta_{-i}^{*}\right)$.

It turns out that the existence of such a stable state is guaranteed the scenario under investigation.

Theorem 3.2: [Existence of an NE for the AF protocol] At least one NE state exists for the PA game described in Sec. I and assuming that the protocol used at the relay is amplify and forward.

Proof: The proof of Theorem 3.2 is based on Theorem 1 of [11]. In [11] Rosen provides sufficient conditions for the existence of an NE in concave games. If, for every player 1) its strategy set is convex and compact, 2) his utility is continuous in the vector of strategies and 3) concave in its own strategy, then the existence of at least one NE is guaranteed. In our setup it is easy to check that conditions 1) and 2) are met. Verifying condition 3 ) for the utility $u_{i}$ is more involving. The second-order derivative of the utility function $u_{i}$ w.r.t. $\theta_{i}$ is intractable because of the term $R_{i}^{(b)}$ which is a function of a rational fraction of $a_{r}\left(\theta_{i}\right)$. It turns out that proving that this second-order derivative is nonpositive is possible if a proper change of variables is made before the sign analysis. This is the purpose of what follows.

First, observe that $R_{i}^{(a)}$ is concave w.r.t. $\theta_{i}$. Thus proving that $R_{i}^{(b)}$ is also concave w.r.t. $\theta_{i}$ will suffice (the sum of two concave functions is concave). We will further consider only user 1 and prove the concavity of $R_{1}^{(b)}$ w.r.t. $\theta_{1}$ (for user 2 the proof is identical). Let us denote $\Phi_{1}\left(\theta_{1}\right)=1+\eta_{1}^{(b)}$. The second derivative of $R_{1}^{(b)}$ w.r.t. $\theta_{1}$ can be written as $\frac{\partial^{2} R_{1}^{(b)}}{\partial \theta_{1}^{2}}=\frac{\Phi_{1}^{\prime \prime} \Phi_{1}-\left(\Phi_{1}^{\prime}\right)^{2}}{\Phi_{1}^{2}}$. In what follows we will show that $\Phi_{1}^{\prime \prime} \Phi_{1}-\left(\Phi_{1}^{\prime}\right)^{2} \leq 0$. For sake of clarity we denote by $\lambda=\rho_{1}\left|h_{11}\right|^{2}, \alpha=\frac{h_{1 r} h_{r 1}}{h_{11}}, \beta=\left|h_{r 1}\right|^{2}, \gamma=h_{21} \sqrt{\theta_{2} \rho_{2}}$, $\delta=h_{2 r} h_{r 1} \sqrt{\theta_{2} \rho_{2}}, \varepsilon_{1}=\sqrt{\frac{\rho_{r}}{\left|h_{2 r}\right|^{2} \theta_{2} \rho_{2}+1}}, \varepsilon_{2}=\frac{\left|h_{1 r}\right|^{2} \rho_{1}}{h_{2 r}^{2} \theta_{2} \rho_{2}+1}$, and we define the function $x\left(\theta_{1}\right)=\varepsilon_{1}\left(1+\varepsilon_{2} \theta_{1}\right)^{-1 / 2}$. We

\footnotetext{
${ }^{1}$ The index $-i$ is the standard notation to refer to the whole set of players except for player $i$.
} 
can now write $\Phi_{1}\left(\theta_{1}\right)=f\left(x\left(\theta_{1}\right)\right)$ with

$$
f(x)=1+\frac{\lambda(1+\alpha x)^{2}\left(\varepsilon_{1}^{2}-x^{2}\right)}{\varepsilon_{2} x^{2}\left[(\gamma+\delta x)^{2}+\beta x^{2}+1\right]} .
$$

The first derivative of $\Phi_{1}$ is given by $\Phi^{\prime}\left(\theta_{1}\right)=\frac{d f}{d x} x^{\prime}$ and the second derivative of $\Phi_{1}$ is given by $\Phi^{\prime \prime}\left(\theta_{1}\right)=$ $\frac{d^{2} f}{d x^{2}}\left(x^{\prime}\right)^{2}+\frac{d f}{d x} x^{\prime \prime}$, where $x^{\prime}$ and $x^{\prime \prime}$ are the first and second order derivatives of $x\left(\theta_{1}\right)$.

Let us define the functions $\mathcal{N}(\cdot)$ and $\mathcal{D}(\cdot)$ that give the numerator and the denominator of a fraction, respectively. One can show that:

$$
\begin{aligned}
\mathcal{N}\left(f^{\prime}(x)\right)= & -2 \lambda x \varepsilon_{2}(1+\alpha x)\left[( \alpha x ^ { 3 } + \varepsilon _ { 1 } ^ { 2 } ) \left((\gamma+\delta x)^{2}\right.\right. \\
& \left.+\beta x^{2}+1\right)+x(1+\alpha x)\left(\varepsilon_{1}^{2}-x^{2}\right) \\
& (\delta(\gamma+\delta x)+\beta x)]
\end{aligned}
$$

and that $\mathcal{D}_{1}(x) \triangleq \mathcal{D}\left(f^{\prime}(x)\right) \quad=$ $\varepsilon_{2}^{2} x^{4}\left[(\gamma+\delta x)^{2}+\beta x^{2}+1\right]^{2}$. We note $\mathcal{N}_{1}(x) \triangleq \mathcal{N}\left(f^{\prime}(x)\right)$, $\mathcal{N}_{0}(x) \triangleq \mathcal{N}(f(x)), \mathcal{D}_{0}(x) \triangleq \mathcal{D}(f(x))$ and then define $\mathcal{N}_{2}(x) \triangleq \frac{d \mathcal{N}_{1}}{d x}$ and $\mathcal{D}_{2}(x) \triangleq \frac{d \mathcal{D}_{1}}{d x}$. Considering all these definitions we further obtain:

$$
\frac{d^{2} f}{d x^{2}}=\frac{\mathcal{N}_{2} \mathcal{D}_{1}-\mathcal{N}_{1} \mathcal{D}_{2}}{\mathcal{D}_{1}^{2}}
$$

and also that:

$\Phi_{1}^{\prime \prime} \Phi-\left(\Phi_{1}^{\prime}\right)^{2}=\frac{\mathcal{N}_{0} \mathcal{N}_{1}\left(\mathcal{D}_{1} x^{\prime \prime}-\mathcal{D}_{2}\left(x^{\prime}\right)^{2}\right)+\left(\mathcal{N}_{0} \mathcal{N}_{2} \mathcal{D}_{1}-\mathcal{N}_{1}^{2} \mathcal{D}_{0}\right)\left(x^{\prime}\right)^{2}}{\mathcal{D}_{0} \mathcal{D}_{1}^{2}}$

To show that $\Phi_{1}^{\prime \prime} \Phi-\left(\Phi_{1}^{\prime}\right)^{2} \leq 0$ it is sufficient to show that both $\Delta_{1} \triangleq \mathcal{D}_{1} x^{\prime \prime}-\mathcal{D}_{2}\left(x^{\prime}\right)^{2}$ and $\Delta_{2} \triangleq \mathcal{N}_{0} \mathcal{N}_{2} \mathcal{D}_{1}-\mathcal{N}_{1}^{2} \mathcal{D}_{0}$ are negative.

One can easily verify that:

$$
\begin{aligned}
\Delta_{1} & =-\frac{\varepsilon_{2}^{2}}{4 \varepsilon_{1}^{4}} x^{9}\left[5\left(\delta^{2}+\beta\right) x^{2}+6 \gamma \delta x+\gamma^{2}+1\right] \\
& {\left[(\gamma+\delta x)^{2}+\beta x^{2}+1\right] } \\
\leq & 0 .
\end{aligned}
$$

Furthermore, it can be shown that $\mathcal{N}_{0} \geq 0, \mathcal{D}_{0} \geq 0, \mathcal{D}_{1} \geq 0$ and that $\mathcal{N}_{2} \leq 0$ and thus $\Delta_{2} \leq 0$. In conclusion $\frac{\partial^{2} R_{1}^{(b)}}{\partial \theta_{1}^{2}} \leq 0$ and thus $R_{1}^{(b)}$ is a concave function of $\theta_{1}$.

What about the uniqueness of the NE? Treating this issue properly is a tough problem and is left as an extension of this paper. In this respect, we will further restrict our attention to special but useful cases. Another reason for analyzing these special cases is that they are less demanding in terms of information required at the transmitters.

\section{B. Case of fixed amplification gains}

Although choosing $a_{r}\left(\theta_{1}, \theta_{2}\right)$ as above allows the relay to exploit all its power, it involves some knowledge on the channels at $\mathcal{S}_{1}, \mathcal{S}_{2}, \mathcal{R}$ and a certain relay structure, which is not always available. In particular, it assumes at the relay the presence of a mechanism to estimate the power of the received signal. While this can be easy for a digital relay transceiver that knows the possible training sequences used by the sources, it might be impossible if the relay is imposed to be a simple analog power amplifier without automatic gain control (AGC). At the sources, the knowledge in terms of channel gains (path losses) depends on the way the PA algorithm is implemented. What matters at this point is that, depending on the amplification gain chosen, the degree of knowledge needed at the sources can be more or less severe. In this section we assume that $a_{r}$ is a certain constant w.r.t. $\underline{\theta}$, denoted by $A_{r}$, that meets the power constraint. In what follows we assume $A_{r}=a_{r}(1,1)$ which is a simple choice meeting the relay power constraint, which can be improved if some statistical information is available. As the case $a_{r}=$ $A_{r}$ is a special case of the general PA game analyzed the existence of an NE is guaranteed thanks to Theorem 3.2.

To study the uniqueness of the NE we exploit the the notion of best responses (BR). The BR of player $i$ to player $j$ is defined by $\operatorname{BR}_{i}\left(\theta_{j}\right)=\arg \max _{\theta_{i}} u_{i}(\underline{\theta})$. In general it is a correspondence but in our case it is just a function. The equilibrium points precisely correspond to the intersection of the BRs of the two users. In this case, using the Lagrangian functions it can be checked that:

$$
\operatorname{BR}_{i}\left(\theta_{j}\right)=\mid \begin{array}{lll}
F_{i}\left(\theta_{j}\right) & , \text { if } & 0<F_{i}\left(\theta_{j}\right)<1 \\
1 & , \text { if } & F_{i}\left(\theta_{j}\right) \geq 1 \\
0 & , \text { otherwise } &
\end{array}
$$

where $j=-i$ and

- $F_{i}\left(\theta_{j}\right) \triangleq-\frac{c_{i j}}{c_{i i}} \theta_{j}+\frac{d_{i}}{c_{i i}}$ is an affine function of $\theta_{j}$;

- for $(i, j) \in\{(1,2),(2,1)\}, c_{i i}=2\left|g_{i i}\right|^{2} \mid A_{r} h_{r i} h_{i r}+$ $\left.h_{i i}\right|^{2} \rho_{i}$;

- $c_{i j}=\left|g_{j i}\right|^{2}\left|A_{r} h_{r i} h_{i r}+h_{i i}\right|^{2} \rho_{j}+\left|g_{i i}\right|^{2} \mid A_{r} h_{r i} h_{j r}+$ $\left.h_{j i}\right|^{2} \rho_{j}$

- $d_{i}=\left|A_{r} h_{r i} h_{i r}+h_{i i}\right|^{2}\left(1+\left|g_{i i}\right|^{2} \rho_{i}+\left|g_{j i}\right|^{2} \rho_{j}\right)-\left|g_{i i}\right|^{2}(1+$ $\left.A_{r}^{2}\left|h_{r i}\right|^{2}\right)$.

If we had no constraints on $\theta_{i}$, then the best responses would be $\operatorname{BR}_{i}\left(\theta_{j}\right)=F_{i}\left(\theta_{j}\right)$, affine functions w.r.t. $\theta_{j}$. In this case we would have a Cournot duopoly [12]. What is very interesting in a Cournot duopoly is that the Cournot tâtonnement process is ensured to converge [13]. As the BRs are affine functions, one is ensured that from any starting state $\left(\theta_{1}^{0}, \theta_{2}^{0}\right)$ this procedure will converge to the unique NE, $\left(\theta_{1}^{\mathrm{NE}}, \theta_{2}^{\mathrm{NE}}\right)=\left(\theta_{1}^{*}, \theta_{2}^{*}\right)$ where

$$
\left\{\begin{aligned}
\theta_{1}^{*} & =\frac{c_{22} d_{1}-c_{12} d_{2}}{c_{11} c_{22}-c_{12} c_{21}} \\
\theta_{2}^{*} & =\frac{c_{11} d_{2}-c_{21} d_{1}}{c_{11} c_{22}-c_{12} c_{21}}
\end{aligned}\right.
$$

represents the unique point of intersection of the two best responses. One has to except of course the particular case where the two lines are superposed $\left(F_{i}\left(\theta_{j}\right)=F_{j}^{-1}\left(\theta_{j}\right)\right)$, where there are an infinity of NE.

However we have to take into consideration the fact that the strategies $\theta_{i} \in[0,1]$ and thus we have to study the best responses given in Eq. (12). It turns out that depending on the parameters $c_{i i} \geq 0, c_{j i} \geq 0, d_{i} \in \mathbb{R}$, there are situations where the intersection of the curves $\theta_{i}=\operatorname{BR}_{i}\left(\theta_{j}\right)$ is not a unique point but a set of two or three different points which correspond to different NE. 
Theorem 3.3: For the game described in Sec. III-A and assuming that the amplification gain at the relay is fixed, $A_{r}=a_{r}(1,1)$, in function of the channel parameters there may be a unique NE, two NE, three NE or an infinity of NE.

Proof: Before discussing these situations in detail, let us first observe that the two functions $F_{i}\left(\theta_{j}\right)$ are decreasing w.r.t. $\theta_{j}$ and also $F_{i}(0)=\frac{d_{i}}{c_{i i}}, F_{i}\left(\theta_{j}^{o}\right)=0$ for $\theta_{j}^{o}=\frac{d_{i}}{c_{i j}}$.

1) If $d_{1} \leq 0$ and $d_{2} \leq 0$ then the BR are constants $\mathrm{BR}_{i}\left(\theta_{j}\right)=0$ and thus the NE is unique $\left(\theta_{1}^{\mathrm{NE}}, \theta_{2}^{\mathrm{NE}}\right)=$ $(0,0)$, for all $c_{i i} \geq 0, c_{j i} \geq 0$.

2) If $d_{1} \leq 0$ and $d_{2}>0$ it can be checked that the NE is unique for all $c_{i i} \geq 0, c_{j i} \geq 0: \theta_{1}^{\mathrm{NE}}=0$ and

$$
\theta_{2}^{\mathrm{NE}}=\mid \begin{array}{lll}
\frac{d_{2}}{c_{22}} & , \text { if } & d_{2}<c_{22} \\
1 & , \text { otherwise. }
\end{array}
$$

3) If $d_{1}>0$ and $d_{2} \leq 0$, similarly to the previous item, we have a unique $\mathrm{NE}$ for all $c_{i i} \geq 0, c_{j i} \geq 0$ : $\theta_{2}^{\mathrm{NE}}=0$ and

$$
\theta_{1}^{\mathrm{NE}}=\mid \begin{array}{lll}
\frac{d_{1}}{c_{11}} & , \text { if } & d_{1}<c_{11} \\
1 & \text {, otherwise. }
\end{array}
$$

4) If $d_{1}>0$ and $d_{2}>0$ we have to consider the other parameters $c_{i i} \geq 0, c_{j i} \geq 0$.

a) If $F_{1}(1) \geq 1$ and $F_{2}(1) \geq 1$ then we have $d_{1} \geq$ $c_{12}+c_{11}$ and $d_{2} \geq c_{21}+c_{22}$. In this case the $\mathrm{BR}$ are constants $\mathrm{BR}_{i}\left(\theta_{j}\right)=1$ and thus the NE is unique $\left(\theta_{1}^{\mathrm{NE}}, \theta_{2}^{\mathrm{NE}}\right)=(1,1)$.

b) If $F_{1}(1) \geq 1$ and $F_{2}(1)<1$ then we have $d_{1} \geq$ $c_{12}+c_{11}$ and $d_{2}<c_{21}+c_{22}$. Here also the NE is unique and $\theta_{1}^{\mathrm{NE}}=1$ and

$$
\theta_{2}^{\mathrm{NE}}=\mid \begin{array}{lll}
\frac{d_{2}-c_{21}}{\alpha_{22}} & , \text { if } & d_{2}>c_{22} \\
0 & , \text { otherwise. }
\end{array}
$$

c) If $F_{1}(1)<1$ and $F_{2}(1) \geq 1$ then we have $d_{1}<$ $c c_{12}+c_{11}$ and $d_{2} \geq c_{21}+c_{22}$. Here also the NE is unique and $\theta_{2}^{\mathrm{NE}}=1$ and

$$
\theta_{1}^{\mathrm{NE}}=\mid \begin{array}{lll}
\frac{d_{1}-c_{12}}{c_{11}} & , \text { if } & d_{1}>c_{11} \\
0 & , \text { otherwise. }
\end{array}
$$

d) If $F_{1}(1)<1$ and $F_{2}(1)<1$ we'll have $d_{1}<$ $c_{12}+c_{11}$ and $d_{2}<c_{21}+c_{22}$. This case is the most demanding one and will be treated in detail separately.

At this point an important observation is in order. The discussed scenarios, for which we have determined the unique $\mathrm{NE}$, have a simple geometric interpretation. If the intersection point $\left(\theta_{1}^{*}, \theta_{2}^{*}\right)$ is such that either $\theta_{1}^{*} \in \mathbb{R} \backslash[0,1]$ or $\theta_{2}^{*} \in \mathbb{R} \backslash[0,1]$ then the NE is unique and differs from this point $\left(\left(\theta_{1}^{\mathrm{NE}}, \theta_{2}^{\mathrm{NE}}\right) \neq\left(\theta_{1}^{*}, \theta_{2}^{*}\right)\right)$. The case 4 .(d) corresponds to the case where the intersection point $\left(\theta_{1}^{*}, \theta_{2}^{*}\right) \in[0,1]^{2}$ is an NE point. Now we are interested in finding whether this intersection point is the unique $\mathrm{NE}$ or are there more than one NE. If $0<d_{1}<c_{11}+c_{12}$ and $0<d_{2}<c_{22}+c_{21}$ we have the following situations:

1) If $c_{11} c_{22}=c_{21} c_{12}$ then the curves described by $\theta_{i}=$ $F_{i}\left(\theta_{j}\right)$ are parallel. a) If $d_{1}=d_{2}$ then the curves are superposed. In this particular case we have an infinity of NE that can be characterized by $\left(\theta_{1}^{\mathrm{NE}}, \theta_{2}^{\mathrm{NE}}\right) \in \mathcal{T}$ where:

$$
\mathcal{T}=\left\{\left(\theta_{1}, \theta_{2}\right) \in[0,1]^{2} \mid \theta_{1}=F_{1}\left(\theta_{2}^{\mathrm{NE}}\right)\right\} .
$$

b) If $d_{1} \neq d_{2}$ then the two lines are only parallel. In this case it can be checked that the NE is unique. In order to explicit the exact relation of the NE, one has to consider all scenarios in function of the sign of the following four relations $F_{i}(0)-1$ and $\theta_{j}^{o}-1$. We will explicit only one of them. Let us assume that $F_{i}(0)-1<0$ and $\theta_{j}^{o}<0$ which means that $d_{1}<\min \left\{c_{12}, \frac{c_{12} c_{21}}{c_{22}}\right.$ and $d_{2}<$ $\min \left\{c_{21}, c_{22}\right\}$. Here we have two sub-cases:

- If $\frac{d_{1}}{c_{12}}<\frac{d_{2}}{c_{22}}$ then the NE is $\theta_{1}^{\mathrm{NE}}=0$ and $\theta_{2}^{\mathrm{NE}}=\frac{d_{2}}{c_{22}}$.

- If $\frac{d_{1}}{c_{12}}>\frac{d_{2}}{c_{22}}$ then the NE is $\theta_{1}^{\mathrm{NE}}=\frac{d_{1} c_{22}}{c_{12} c_{21}}$ and $\theta_{2}^{\mathrm{NE}}=0$.

2) If $c_{11} c_{22} \neq c_{21} c_{12}$. Here we have to consider all cases in function of the sign of the four relations $F_{i}(0)-1$ and $\theta_{j}^{o}-1$. We will focus on only one of them. Let us assume that $F_{i}(0)-1<0$ and $\theta_{j}^{o}<0$ and thus $d_{1}<\min \left\{c_{12}, c_{11}\right\}$ and $d_{2}<\min \left\{c_{21}, c_{22}\right\}$. Here we have four sub-cases:

- If $\frac{d_{2}}{c_{22}}<\frac{d_{1}}{c_{12}}$ and $\frac{d_{1}}{c_{11}}>\frac{d_{2}}{c_{21}}$ then the NE is unique $\theta_{1}^{\mathrm{NE}}=\theta_{1}^{*}$ and $\theta_{2}^{\mathrm{NE}}=\theta_{2}^{*}$,

- If $\frac{d_{2}}{c_{22}}>\frac{d_{1}}{c_{12}}$ and $\frac{d_{1}}{c_{11}}<\frac{d_{2}}{c_{21}}$ then there are three different NE

$\left(\theta_{1}^{\mathrm{NE}}, \theta_{2}^{\mathrm{NE}}\right) \in\left\{\left(\theta_{1}^{*}, \theta_{2}^{*}\right),\left(0, \frac{d_{2}}{c_{22}}\right),\left(\frac{d_{1}}{c_{11}}, 0\right)\right\}$.

- If $\frac{d_{2}}{c_{22}}=\frac{d_{1}}{c_{12}}$ and $\frac{d_{1}}{c_{11}}<\frac{d_{2}}{c_{21}}$ then there are only two different NE $\left(\theta_{1}^{\mathrm{NE}}, \theta_{2}^{\mathrm{NE}}\right) \in\left\{\left(0, \frac{d_{2}}{c_{22}}\right),\left(\frac{d_{1}}{c_{11}}, 0\right)\right\}$.

- If $\frac{d_{2}}{c_{22}}>\frac{d_{1}}{c_{12}}$ and $\frac{d_{1}}{c_{11}}=\frac{d_{2}}{c_{21}}$ then there are two NE $\left(\theta_{1}^{\mathrm{NE}}, \theta_{2}^{\mathrm{NE}}\right) \in\left\{\left(\frac{d_{1}^{c_{11}}}{c_{11}}, 0\right),\left(0, \frac{d_{2}}{c_{22}}\right)\right\}$.

In conclusion, the cases where there are multiple $\mathrm{NE}$ are: 1) either when the lines $\theta_{i}=F_{i}\left(\theta_{j}\right)$ are superposed and the game has an infinity of NE or 2). when the lines have a unique intersection point $\left(\theta_{1}^{*}, \theta_{2}^{*}\right)$ that lies inside $[0,1] \times[0,1]$. In the latter cases there can be one, two or three different NE.

The cases with an infinity of NE and those with two NE can be proven to happen with probability zero when considering random channel gains with continuous probability distributions. The NE selection issue however is beyond the scope of this paper. A possible way to tackle this problem would to consider hierarchical games, where one user is assumed to be the leader of the game and chooses hits best strategy having complete information on the strategy chosen by its opponent [15].

So far we have considered that the amplification gain was fixed at a certain value. Clearly, the value $a_{r}(1,1)$ is feasible in terms of power constraint but is that the best choice in terms of achievable rates? Of one the weaknesses of the AF protocol is that it also amplifies the noise received by the relay. Indeed, some authors have shown that saturating the power constraint at the relay is not always optimal [16], 
[17], [18], [19] in a sense of certain performance metrics. For example, the authors of [16] derived the best relaying function in the sense of the raw bit error rate when no direct link is assumed and a BPSK modulation is used at the source and relay. In [17] an optimized relaying function in the sense of the mutual information assuming that there is no direct link is proposed. In [18] the authors study the best relaying function in the sense of the minimum square error for the frequency division relay channel and in [19] the authors discuss the choice of the optimal amplification gain w.r.t. the achievable rate and show that it is not always the one saturating the relay power constraint and it strongly depends on the channel parameters.

Here, we formulate the problem as a Stackelberg game where the relay becomes a player and more precisely the leader of the game. His strategy is the amplification gain $A_{r} \in\left[0, a_{r}\left(\theta_{1}, \theta_{2}\right)\right] ;$ the source nodes are therefore the followers of the game. We consider two choices for the leader's utility: A) the rate of a given user (1 or 2), B) the system sum-rate $u(A r)=u_{1}\left[\underline{\theta}^{\mathrm{NE}}(A r)\right]+u_{2}\left[\underline{\theta}^{\mathrm{NE}}(A r)\right]$. Choice A) would correspond to the case where one of the users would be able to choose the amplification gain whereas B) would correspond, for example, to the case where the system is owned by the same provider or where an agreement between two providers would have been found. The sources (the followers of the game) react to the leader's strategy by choosing their best selfish PA policies. Interestingly, we know from Theorem 3.2 that for any location of the relay there will be an equilibrium. The goal of the leader is to make this equilibrium efficient in the sense of $\mathrm{A}$ ) or $\mathrm{B}$ ).

The first question we ask is whether there exists such an amplification gain that maximizes the achievable transmission rates without saturating the relay power constraint. For a simple relay channel it is easy to check that, depending on the channel parameters, it is not always optimal to satisfy the power constraint at the relay.

Let us consider the first situation where the leader chooses the relay amplification factor that maximizes the transmission rate of user $i \in\{1,2\}$. We assume that the power allocations of the two users are fixed (e.g., an NE point), $\left(\tilde{\theta}_{1}, \tilde{\theta}_{2}\right)$. It is straightforward to see that it will choose $A_{r}^{*}$ such that $A_{r}^{*}=\arg \max _{A_{r} \in\left[0, \bar{a}_{r}\right]} R_{i}^{(b)}\left(A_{r}\right)$, with $\bar{a}_{r}=a_{r}\left(\tilde{\theta}_{1}, \tilde{\theta}_{2}\right)$.

Theorem 3.4: [Optimal amplification gain] The transmission rate of user $i$ in the IRC, $R_{i}^{(b)}\left(A_{r}\right)$, as a function of $A r \in\left[0, \bar{a}_{r}\right]$, has two critical points: $A_{r, i}^{(1)}=-\frac{n_{i}}{m_{i}}$ and $A_{r, i}^{(2)}=-\frac{m_{i} q_{i}^{2}+m_{i}-p_{i} q_{i} n_{i}}{m_{i} q_{i} p_{i}-p_{i}^{2} n_{i}-n_{i} s_{i}}$, where $m_{i}=h_{i r} h_{r i} \sqrt{\rho_{i} \tilde{\theta}_{i}}$, $n_{i}=h_{i i} \sqrt{\rho_{i} \tilde{\theta}_{i}}, p_{i}=h_{j r} h_{r i} \sqrt{\rho_{j} \tilde{\theta}_{j}}, q_{i}=h_{j i} \sqrt{\rho_{j} \tilde{\theta}_{j}}$, $s_{i}=h_{r i}^{2}, \bar{a}_{r}=a_{r}\left(\tilde{\theta}_{1}, \tilde{\theta}_{2}\right)$ and $j=-i$. Thus the optimal amplification gain, $A_{r}^{*}=\arg \max _{A_{r} \in\left[0, \bar{a}_{r}\right]} R_{i}^{(b)}\left(A_{r}\right)$, depending on the channel parameters it takes a value in the set $A_{r}^{*} \in$ $\left\{0, \bar{a}_{r}, A_{r, i}^{(1)}, A_{r, i}^{(2)}\right\}$.

Proof: Using the notations given in Theorem 3.4 and also Eq. (8) the rate $R_{i}^{(b)}$ can be written as:

$$
R_{i}^{(b)}\left(A_{r}\right)=\log \left(1+\frac{\left(m_{i} A_{r}+n_{i}\right)^{2}}{\left(p_{i} A_{r}+q_{i}\right)^{2}+s_{i} A_{r}^{2}+1}\right),
$$

We observe that $R_{i}^{(b)}(0)=\log \left(1+\frac{n_{i}^{2}}{q_{i}^{2}+1}\right)$ and that we have a horizontal asymptote

$R_{i, \infty}^{(b)} \triangleq \lim _{A_{r} \rightarrow \infty} R_{1}^{(b)}\left(A_{r}\right)=\left(1+\frac{m_{i}^{2}}{p_{i}^{2}+s_{i}}\right)$. Also the first derivative w.r.t. $A_{r}$ is

$$
R_{i}^{(b)^{\prime}}\left(A_{r}\right)=
$$

$\frac{\left(m_{i} A_{r}+n_{i} s_{i}\right)\left[\left(m_{i} q_{i} p_{i}-p_{i}^{2} n_{i}-n_{i} s_{i}\right) A_{r}+m_{i} q_{i}^{2}+m_{i}-n_{i} q_{i} p_{i}\right]}{\left[\left(p_{i} A_{r}+q_{i}\right)^{2}+s_{i} A_{r}^{2}+1\right]\left[\left(m_{i} A_{r}+n_{i}\right)^{2}+\left(p_{i} A_{r}+q_{i}\right)^{2}+s_{i} A_{r}^{2}+1\right]}$

It is straightforward to see that the critical points are: $A_{r, i}^{(1)}=$ $-\frac{n_{i}}{m_{i}}$ and $A_{r, i}^{(2)}=-\frac{m_{i} q_{i}^{2}+m_{i}-p_{i} q_{i} n_{i}}{m_{i} q_{i} p_{i}-p_{i}^{2} n_{i}-n_{i} s_{i}}$.

The explicit solution, $A_{r}^{*}$ depends on the channel parameters and is given below.

1) If $m_{i}\left(m_{i} q_{i} p_{i}-p_{i}^{2} n_{i}-n_{i}\right) \geq 0$ then

a) if $A_{r, i}^{(1)} \leq 0$ and $A_{r, i}^{(2)} \leq 0$ then $A_{r}^{*}=\bar{a}_{r}$;

b) if $A_{r, i}^{(1)}>0$ and $A_{r, i}^{(2)} \leq 0$ then

i) if $\bar{a}_{r} \geq A_{r, i}^{(1)}$ then $A_{r}^{*}=0$;

ii) if $\bar{a}_{r}<A_{r, i}^{(1)}$ then

- if $R_{i}^{(b)}(0) \geq R_{i}^{(b)}\left(\bar{a}_{r}\right)$ then $A_{r}^{*}=0$;

- if $R_{i}^{(b)}(0)<R_{i}^{(b)}\left(\bar{a}_{r}\right)$ then $A_{r}^{*}=\bar{a}_{r}$;

c) if $A_{r, i}^{(1)} \leq 0$ and $A_{r, i}^{(2)}>0$ then the analysis is similar to the previous case and $A_{r}^{*} \in\left\{0, \bar{a}_{r}\right\}$ depending on $A_{r}^{(2)}$ this time;

d) if $A_{r, i}^{(1)}>0$ and $A_{r, i}^{(2)}>0$

i) if $A_{r, i}^{(1)}<A_{r, i}^{(2)}$

A) if $\bar{a}_{r} \leq A_{r, i}^{(1)}$ then $A_{r}^{*}=\bar{a}_{r}$;

B) if $A_{r, i}^{(1)}<\bar{a}_{r} \leq A_{r, i}^{(2)}$ then $A_{r}^{*}=A_{r, i}^{(1)}$;

C) if $\bar{a}_{r}>A_{r, i}^{(2)}$ then

- if $R_{i}^{(b)}\left(A_{r, i}^{(1)}\right) \geq R_{1}^{(b)}\left(\bar{a}_{r}\right)$ then $A_{r}^{*}=$ $A_{r, i}^{(1)}$;

- if $R_{i}^{(b)}\left(A_{r, i}^{(1)}\right)<R_{1}^{(b)}\left(\bar{a}_{r}\right)$ then $A_{r}^{*}=$ $\bar{a}_{r}$

ii) if $A_{r, i}^{(1)}>A_{r, i}^{(2)}$ then the analysis is similar to the previous case, exchanging the roles of $A_{r, i}^{(1)}$ and $A_{r i}^{(2)}$;

iii) if $A_{r, i}^{(1)}=A_{r, i}^{(2)}$ then $A_{r}^{*}=\bar{a}_{r}$.

2) If $m_{i}\left(m_{i} q_{i} p_{i}-p_{i}^{2} n_{i}-n_{i}\right)<0$ then

a) if $A_{r, i}^{(1)} \leq 0$ and $A_{r, i}^{(2)} \leq 0$ then $A_{r}^{*}=0$;

b) if $A_{r, i}^{(1)}>0$ and $A_{r, i}^{(2)} \leq 0$ then

i) if $\bar{a}_{r} \geq A_{r, i}^{(1)}$ then $A_{r}^{*}=\bar{a}_{r}$;

ii) if $\bar{a}_{r}>A_{r, i}^{(1)}$ then $A_{r}^{*}=A_{r, i}^{(1)}$;

c) if $A_{r, i}^{(1)} \leq 0$ and $A_{r, i}^{(2)}>0$ then the analysis is similar to the previous case and $A_{r}^{*} \in\left\{\bar{a}_{r}, A_{r, i}^{(1)}\right\}$ depending on $A_{r, i}^{(2)}$ this time; 
d) if $A_{r, i}^{(1)}>0$ and $A_{r, i}^{(2)}>0$

i) if $A_{r, i}^{(1)}<A_{r, i}^{(2)}$

A) if $\bar{a}_{r} \leq A_{r, i}^{(1)}$ then $A_{r}^{*}=0$;

B) if $A_{r, i}^{(1)}<\bar{a}_{r} \leq A_{r, i}^{(2)}$ then

- if $R_{i}^{(b)}(0) \geq R_{i}^{(b)}\left(\bar{a}_{r}\right)$ then $A_{r}^{*}=0$;

- if $R_{i}^{(b)}(0)<R_{i}^{(b)}\left(\bar{a}_{r}\right)$ then $A_{r}^{*}=\bar{a}_{r}$;

C) if $\bar{a}_{r}>A_{r, i}^{(2)}$ then

- if $R_{i}^{(b)}\left(A_{r, i}^{(2)}\right) \geq R_{i}^{(b)}(0)$ then $A_{r}^{*}=$ $A_{r, i}^{(2)}$

- if $R_{i}^{(b)}\left(A_{r, i}^{(2)}\right)<R_{i}^{(b)}(0)$ then $A_{r}^{*}=0$;

ii) if $A_{r, i}^{(1)}>A_{r, i}^{(2)}$ then the analysis is similar to the previous case, exchanging the roles of $A_{r, i}^{(1)}$ and $A_{r, i}^{(2)}$;

iii) if $A_{r, i}^{(1)}=A_{r, i}^{(2)}$ then $A_{r}^{*}=0$.

We observe that, depending on the channel parameters, it is not always optimal to saturate the power constraint at the relay.

Now we consider the case where the strategy of the leader is to choose the amplification gain that maximizes the sumrate of the network. It is straightforward to see that it will choose $A_{r}^{*}=\arg \max \left\{R_{1}^{(b)}\left(A_{r}\right)+R_{2}^{(b)}\left(A_{r}\right)\right\}$. In order to simplify the analysis we use similar notations as the previous case. Let us denote by $R_{1}^{(b)}\left(A_{r}\right)+R_{2}^{(b)}\left(A_{r}\right)=E\left(A_{r}\right)$

$$
E\left(A_{r}\right)=\sum_{i=1}^{2} \log \left(1+\frac{\left(m_{i} A_{r}+n_{i}\right)^{2}}{\left(p_{i} A_{r}+q_{i}\right)^{2}+s_{i} A_{r}^{2}+1}\right),
$$

w.r.t. $A_{r} \in\left[0, \bar{a}_{r}\right]$ where the parameters are identical to the ones defined in Theorem 3.4. We observe that

$$
E(0)=\log \left(1+\frac{n_{1}^{2}}{q_{1}^{2}+1}\right)+\log \left(1+\frac{n_{2}^{2}}{q_{2}^{2}+1}\right)
$$

and that we have a horizontal asymptote

$$
E_{\infty} \triangleq \lim _{A_{r} \rightarrow \infty} E\left(A_{r}\right)=\left(1+\frac{m_{1}^{2}}{p_{1}^{2}+q_{1}}\right)+\left(1+\frac{m_{2}^{2}}{p_{2}^{2}+q_{2}}\right) .
$$

In this case, it is not easy to obtain closed-form expressions of the optimal amplification gain (in order to find the critical points of $E$ one has to solve a six degree equation). This is why numerical results will be provided to illustrate the fact it is not always optimal to saturate the relay power constraint.

\section{WHEN THE RELAY IMPLEMENTS THE DF PROTOCOL}

In this section we consider a different relaying protocol, the decode-and-forward scheme [9]. Here, the relay decodes the messages sent by the two information sources. In Gaussian relay channels, this protocol can lead to better performance in terms of achievable rates than the $\mathrm{AF}$ and $\mathrm{EF}$ protocols; this typically occurs in practice when the relay is close to the source in comparison with the destination [14]. The principle of the DF protocol is detailed in [9] and here we just give the main idea behind it. Consider a Gaussian relay channel for which the source-relay link has a better quality than the source-destination link. From each information message, the source builds a coarse and a fine message. With these two messages the source superposes two codewords. The rates associated with these codewords (or messages) are such that the relay can decode both of them reliably while the destination can only decode the coarse message. After decoding this message, the destination can subtract the corresponding signal and try to decode the fine message. To help the destination to do so, the relay cooperates with the source by sending some information about the fine message. We will see a little further that the cooperation degree between the source and relay can be measured in terms of correlation between two random variables. The intuition being that if the source only sends a fine message, the cooperation degree is 1 while if it sends only a coarse message, it is 0 . The transmission rate over the relay channel is maximized, in general, for an intermediate value of the cooperation degree.

The received baseband signals write

$$
\left\{\begin{array}{l}
Y_{r}^{(b)}=h_{1 r} X_{1}^{(b)}+h_{2 r} X_{2}^{(b)}+Z_{r} \\
Y_{1}^{(b)}=h_{11} X_{1}^{(b)}+h_{r 1} X_{r 1}^{(b)}+h_{21} X_{2}^{(b)}+h_{r 1} X_{r 2}^{(b)}+Z_{1}^{(b)} \\
Y_{2}^{(b)}=h_{22} X_{2}^{(b)}+h_{r 2} X_{r 2}^{(b)}+h_{12} X_{1}^{(b)}+h_{r 2} X_{r 1}^{(b)}+Z_{2}^{(b)}
\end{array}\right.
$$

where $X_{r 1}^{(b)} \sim \mathcal{N}\left(0, \nu P_{r}\right), X_{r 2}^{(b)} \sim \mathcal{N}\left(0,(1-\nu) P_{r}\right)$ and the other power constraints are given in Sec. II. The parameter $\nu \in[0,1]$ is chosen by the relay to share its available power between the codewords that it transmits to the two receivers. The achievable rates region on the IRC is given by:

$$
\left\{\begin{aligned}
R_{1}^{(b)} & \leq \min \left\{\begin{array}{c}
I\left(X_{1}^{(b)} ; Y_{r} \mid X_{2}^{(b)}, X_{r 1}^{(b)}, X_{r 2}^{(b)}\right), \\
I\left(X_{1}^{(b)}, X_{r 1}^{(b)} ; Y_{1}^{(b)}\right)
\end{array}\right\} \\
R_{2}^{(b)} & \leq \min \left\{\begin{array}{c}
I\left(X_{2}^{(b)} ; Y_{r} \mid X_{1}^{(b)}, X_{r 1}^{(b)}, X_{r 2}^{(b)}\right) \\
I\left(X_{2}^{(b)}, X_{r 2}^{(b)} ; Y_{2}^{(b)}\right)
\end{array}\right\} \\
R_{1}^{(b)}+R_{2}^{(b)} & \leq I\left(X_{1}^{(b)}, X_{2}^{(b)} ; Y_{r} \mid X_{r 1}, X_{r 2}\right),
\end{aligned}\right.
$$

where the first terms in the first two inequalities and the third inequality correspond to the decoding constraint at the relay. Note that the transmission between both source nodes and the relay is equivalent to a Multiple Access Channel (MAC) for which the capacity region is fully characterized. Here we assume that the destination implements single-user decoding. There are many motivations for this choice, here we just give two of them. It is a realistic assumption in a framework where devices operate in unlicensed band in a priori noncoordinated manner. It allows us to cope with the constraint on the MAC sum-rate, which would make the game more complex to be played and is therefore left as an extension of this paper. The corresponding rate pair is characterized by the fact that, in the decoding step of a source's message at the relay, the other source's signal is treated as additive noise. Therefore, a rate pair achievable by the IRC is given by 


$$
\left\{\begin{array}{l}
R_{1}^{(b)}=\min \{\underbrace{I\left(X_{1}^{(b)} ; Y_{r} \mid X_{r 1}^{(b)}, X_{r 2}^{(b)}\right)}_{R_{1,1}^{(b)}}, \underbrace{I\left(X_{1}^{(b)}, X_{r 1}^{(b)} ; Y_{1}^{(b)}\right)}_{R_{1,2}^{(b)}}\} \\
R_{2}^{(b)}=\min \{\underbrace{I\left(X_{2}^{(b)} ; Y_{r} \mid X_{r 1}^{(b)}, X_{r 2}^{(b)}\right)}_{R_{2,1}^{(b)}}, \underbrace{I\left(X_{2}^{(b)}, X_{r 2}^{(b)} ; Y_{2}^{(b)}\right)}_{R_{2,2}^{(b)}}\}
\end{array}\right.
$$

where

$$
\left\{\begin{aligned}
R_{1,1}^{(b)} & =\mathcal{C}\left(\frac{h_{1 r}^{2}\left(1-\tau_{1}\right) \theta_{1} P_{1}}{h_{2 r}^{2}\left(1-\tau_{2}\right) \theta_{2} P_{2}+N_{r}}\right) \\
R_{2,1}^{(b)} & =\mathcal{C}\left(\frac{h_{2 r}^{2}\left(1-\tau_{2}\right) \theta_{2} P_{2}}{h_{1 r}^{2}\left(1-\tau_{1}\right) \theta_{1} P_{1}+N_{r}}\right) \\
R_{1,2}^{(b)} & =\mathcal{C}\left(\frac{h_{11}^{2} \theta_{1} P_{1}+h_{r 1}^{2} \nu P_{r}+2 h_{11} h_{r 1} \sqrt{\tau_{1} \theta_{1} P_{1} \nu P_{r}}}{h_{21}^{2} \theta_{2} P_{2}+h_{r 1}^{2} \bar{\nu} P_{r}+2 h_{21} h_{r 1} \sqrt{\tau_{2} \theta_{2} P_{2} \bar{\nu} P_{r}}+N_{1}^{(b)}}\right) \\
R_{2,2}^{(b)} & =\mathcal{C}\left(\frac{h_{22}^{2} \theta_{2} P_{2}+h_{r 2}^{2} \bar{\nu} P_{r}+2 h_{22} h_{r 2} \sqrt{\tau_{2} \theta_{2} P_{2} \bar{\nu} P_{r}}}{h_{12}^{2} \theta_{1} P_{1}+h_{r 2}^{2} \nu P_{r}+2 h_{12} h_{r 2} \sqrt{\tau_{1} \theta_{1} P_{1} \nu P_{r}}+N_{2}^{(b)}}\right)
\end{aligned}\right.
$$

with $\left(\nu, \tau_{1}, \tau_{2}\right) \in[0,1]^{3}, i \in\{1,2\}$, and $\bar{\nu}=1-\nu$. In the coding strategy used to achieve these rates, the cooperation degree between the source node $\mathcal{S}_{i}$ and relay node is defined as follows:

$$
\tau_{i}=\frac{E\left[X_{i}^{(b)} X_{r i}^{(b) *}\right]}{\sqrt{E\left[\left|X_{i}^{(b)}\right|^{2}\right] E\left[\left|X_{r i}^{(b)}\right|^{2}\right]}} .
$$

In fact we can rewrite $X_{i}^{(b)}$ as $X_{i}^{(b)}=X_{i 0}^{(b)}+\frac{\tau_{i} P_{i}}{\nu_{i} P_{r}} X_{r i}$ where the codewords $X_{i 0}^{(b)}$ and $X_{r i}$ are independent with $X_{i 0}^{(b)} \sim$ $\mathcal{N}\left(0,\left(1-\tau_{i}\right) P_{i}\right)$ and $\nu_{1}=\nu$ and $\nu_{2}=\bar{\nu}$; the first term $X_{i 0}^{(b)}$ represents the coarse message while the second term corresponds to the fine message. In comparison to the AF case, these cooperation degrees represent a supplementary degree of freedom that each source node can use in the maximization of its individual rate. The achievable rates on the IC are given in Sec. II and the global achievable rates at the receiver nodes are therefore given by $R_{i}=R_{i}^{(a)}+R_{i}^{(b)}$.

\section{A. General power allocation game}

As we have mentioned in the previous section, the cooperation degree $\tau_{i}$, has to be considered. If we assume that these parameters are chosen at the transmitters then the interaction between the two users is characterized by both: their power allocation policies between the the IC and IRC $\theta_{i}$ and also by their cooperation degrees with the relay node $\tau_{i}$. In this case we formulate the game in the following way.

- the players of the game are the two transmitters;

- the strategy of transmitter $i$ consists in choosing the vector $\left(\theta_{i}, \tau_{i}\right)$ in its strategy set $\mathcal{A}_{i}=[0,1] \times[0,1]$ in order to maximize its individual transmission rate $R_{i}$;

- the utility function for user $i \in\{1,2\}$ is its achievable Shannon transmission rate on both frequency bands given by $u_{i}\left(\left(\theta_{i}, \tau_{i}\right),\left(\theta_{-i}, \tau_{-i}\right)\right)=R_{i}^{(a)}+R_{i}^{(b)}$, where $R_{i}^{(b)}$ are the transmission rates defined in Eq. (15).

In this case the state of the network becomes the quadruplet $\left(\theta_{1}, \tau_{1}, \theta_{2}, \tau_{2}\right)$. The existence of the $\mathrm{NE}$ is more difficult to be dealt with. We will have to consider more advanced mathematical tools to prove the existence in this case. In what follows we focus on two particular cases: the case of fixed cooperation degrees and the case of fixed power allocation policies among the two channels.

\section{B. Case of fixed cooperation degrees}

In this case we assume that the cooperation degrees $\left(\tau_{1}, \tau_{2}\right)$ are fixed and only the power allocation policy can be optimized by the sources. This assumption is realistic if we consider that not the transmitters but the relay has the control over these cooperation degrees (the MAC $\left(\mathcal{S}_{1}, \mathcal{S}_{2}\right)-\mathcal{R}$ can be view as a centralized network in which the relay node chooses the operating point on the MAC capacity region). The users react accordingly to the choice of the relay node. Now, for user $i$, the remaining degree of freedom in the maximization of its individual rate is the power fraction $\theta_{i}$ that it allocates to the IRC. The game is a power allocation game very similar to the AF case. It turns out that in this case the existence of the equilibrium is guaranteed.

Theorem 4.1: [Existence of an NE for the DF protocol] There will always be an NE in the power allocation game described assuming that the relaying protocol is decode-andforward and that the cooperation degrees are fixed.

Proof: The proof of this theorem is also based on Theorem 1 of [11]. In contrast with the case of AF, the utility function of the user $i$ is the minimum between two rates. Hence, proving the concavity of these two rate functions w.r.t. $\theta_{i}$ implies the concavity of $u_{i}$ (the $\min$ function of two concave functions is also concave).

The uniqueness issue is however more difficult to be dealt with properly for the same reasons given for the general power allocation game with the AF protocol.

Due to the fact that the relay chooses the cooperation degrees, we can propose a Stackelberg formulation to model the game, similar to Sec. III-B. The relay is the leader of the game and it can tune its strategy $\left(\tau_{1}, \tau_{2}\right)$ to optimize a certain performance criteria.

\section{Case of fixed power allocation policies}

We now suppose that the power fractions $\theta_{1}$ and $\theta_{2}$ allocated to the IRC are fixed (for example $\theta_{1}=\frac{1}{2}$ and $\theta_{2}=\frac{1}{2}$ ). This scenario is realistic if there is not a feedback mechanism that could help the sources to obtain the interference levels their receivers see on band (a). Assuming the presence of such a mechanism on band (b) is more realistic due to the presence of the relay node that could manage the CSI acquisition. For user $i$, the remaining degree of freedom in the maximization of its individual rate is its cooperation degree $\tau_{i}$ with the relay node. We want to know if there exists an NE for this PA game. The answer is given in the theorem below.

Theorem 4.2: [Existence of an NE for the DF protocol] At least one NE exists in the power allocation game assuming the decode-and-forward protocol and also that the power allocation policies over the two channels are fixed.

Proof: The proof of this theorem also relies on Theorem 1 of [11]. It is easy to check that, for the user $i$, the two rates 
functions in the utility function are concave w.r.t. $\tau_{i}$ hence the utility function $u_{i}$ is also concave w.r.t. $\tau_{i}$.

Just like in the previous case, the uniqueness issue is however more difficult to be dealt with properly. As an extension of the general power allocation, we could consider a Stackelberg formulation for which the relay node is the leader of the game and it chooses its power sharing factor $\nu \in[0,1]$ to maximize a certain performance criteria. The users will react accordingly to the value of this parameter.

\section{NUMERICAL RESULTS}

In this section we focus our attention on the amplify-andforward relaying protocol, the case where the amplification gain is constant. Also we assume that the channel gains $g_{i j}$ and $h_{i j}, i, j \in\{1,2\}$ are real variables.

First, we study the best response functions defined in Sec. III-B (see Eq. 12) to illustrate the theoretical analysis we conducted for the determination of the possible equilibria states. In Fig. 2 we plot the best response functions for the following scenario: $\rho_{1}=1, \rho_{2}=3, \rho_{r}=2$ and the channel gains $\left(g_{11}, g_{12}, g_{21}, g_{22}\right)=(-1.7,4.31,8.35,1.37)$, $\left(h_{11}, h_{12}, h_{21}, h_{22}\right)=(1.89,4.72,0.2,-0.2) \quad$ and $\left(h_{1 r}, h_{2 r}, h_{r 1}, h_{r 2}\right)$

$=(-2.5,3.23,5.58,3.77)$. The intersection point of the curves $F_{i}\left(\theta_{j}\right)=\theta_{i}$ is $\left(\theta_{1}^{*}, \theta_{2}^{*}\right)=(0.84,0.66) \in[0,1]^{2}$ and thus is an NE point. We further observe that this point is not the only equilibrium and that there are two more NE: $(0,1)$, $(1,0.37)$. In Fig. 3 we plot the best response functions for the following scenario: $\rho_{1}=1, \rho_{2}=3, \rho_{r}=2$ and the channel gains $\left(g_{11}, g_{12}, g_{21}, g_{22}\right)=(5.29,2.89,3.36,-1.16)$, $\left(h_{11}, h_{12}, h_{21}, h_{22}\right)=(3.79,2.54,0.38,6.55)$ and $\left(h_{1 r}, h_{2 r}, h_{r 1}, h_{r 2}\right)=(-3.18,1.67,-1.11,1.25)$. The intersection point of the curves $F_{i}\left(\theta_{j}\right)=\theta_{i}$ is $\left(\theta_{1}^{*}, \theta_{2}^{*}\right)=$ $(0.25,1,39) \notin[0,1]^{2}$ and thus it is not an equilibrium. In this case the NE is unique and given by $\left(\theta_{1}^{\mathrm{NE}}, \theta_{2}^{\mathrm{NE}}\right)=(0.49,1)$.

Second, we consider the maximization of the overall network sum-rate as a function of the amplification gain at the relay. In Fig. 4 we plot the sum $E\left(A_{r}\right)=$ $R_{1}^{(b)}+R_{2}^{(b)}$ as a function of $A_{r} \in\left[0, \bar{a}_{r}\right]$ for the fixed power allocation policies $\left(\theta_{1}, \theta_{2}\right)=(0.5,0.5)$, $\rho_{1}=1, \rho_{2}=3, \rho_{r}=2$ and the channel gains $\left(h_{11}, h_{12}, h_{21}, h_{22}\right)=(5.51,1.53,0.36,-0.87)$ and $\left(h_{1 r}, h_{2 r}, h_{r 1}, h_{r 2}\right)=(-2.63,2.91,-1.96,2.44)$ and fixed $\left(\tilde{\theta}_{1}, \tilde{\theta}_{2}\right)=(0.5,0.5)$. We observe that choosing the amplification gain equal to $\bar{a}_{r}=0.34$ (saturating the constraint) is not optimal and $A_{r}^{*}=0.04<\bar{a}_{r}$.

\section{CONCLUSION}

In this paper we have introduced a channel model which is very useful to study scenarios where each wireless device operating on unlicensed bands is offered the opportunity to exploit additional resources to increase its own transmission rate. Here the additional resources consist of spectrum plus cooperation power. We have analyzed in detail the problem of decentralized power allocation for two important classes of relaying protocols (AF and DF) and shown the existence of predictable states for the network. This is very interesting

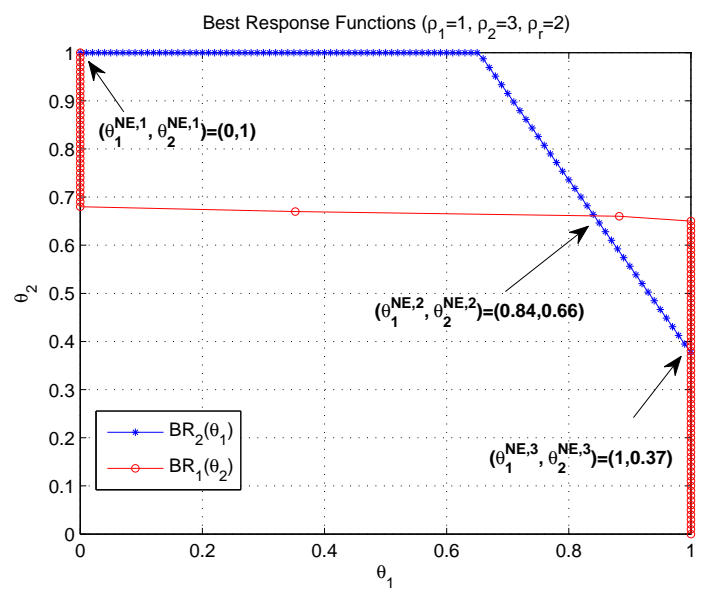

Fig. 2. Amplify-and-Forward, case of fixed amplification gain. The Best Response Functions. The intersection point $\left(\theta_{1}^{*}, \theta_{2}^{*}\right) \in[0,1]^{2}$. There are three different NE points.

since it shows how selfish users self-regulate to reach an equilibrium. Unfortunately, we have seen that even for the simple case of the AF protocol, the equilibrium is not unique. The effective equilibrium reached after Cournot tâtonnement depends on the network initial state. This shows the interest in designing physical mechanisms to give incentive to users to converge towards a given equilibrium or finding agreements between operators and manufacturers deploying wireless devices in a non-coordinated manner. We have also seen that the DF protocol naturally introduces a game, even when the power allocation policy is fixed at each source, through the concept of cooperation degree between the sources and relay. At last we have seen that our problem can also be formulated as a Stackelberg game when some key parameters need to be optimized namely: the amplification gain used by the relay when $\mathrm{AF}$ is assumed, the power allocation at the relay (between the two relayed signals), the relay location. In these cases, the relay becomes a player of the game (leader). We have not treated all these issues here for obvious reasons of space but we already know that the best strategy of the relay is not to transmit with its maximum available power. There is a certain value of the amplification gain that corresponds to the optimum trade-off between cooperation and noise amplification and maximize its utility (e.g., individual transmission rate of a user or system sum-rate). From a broader perspective, many extensions of this work have been proposed, which should help to better analyze the general problem of competition in cooperative channels.

\section{REFERENCES}

[1] "First Report and Order for Ultra Wideband Technology", Federal Communications Commision, 14 Feb. 2002.

[2] "Report of the Spectrum Efficiency Working Group", Federal Communications Commision, Nov. 2002.

[3] A. B. Carleial, "Interference Channels", IEEE Trans. on Inform. Theory, Vol. 24, No. 1, pp. 66-70, Jan. 1978. 


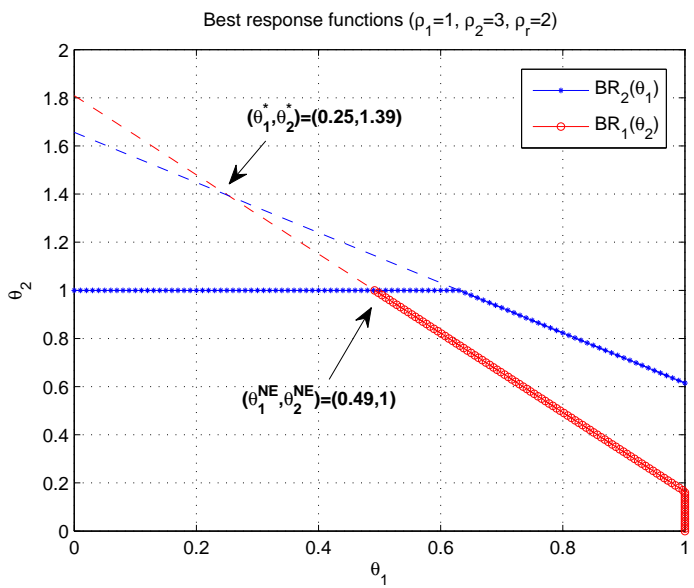

Fig. 3. Amplify-and-Forward, case of fixed amplification gain. The Best Response Functions. The intersection point $\left(\theta_{1}^{*}, \theta_{2}^{*}\right) \notin[0,1]^{2}$. There is a unique NE.

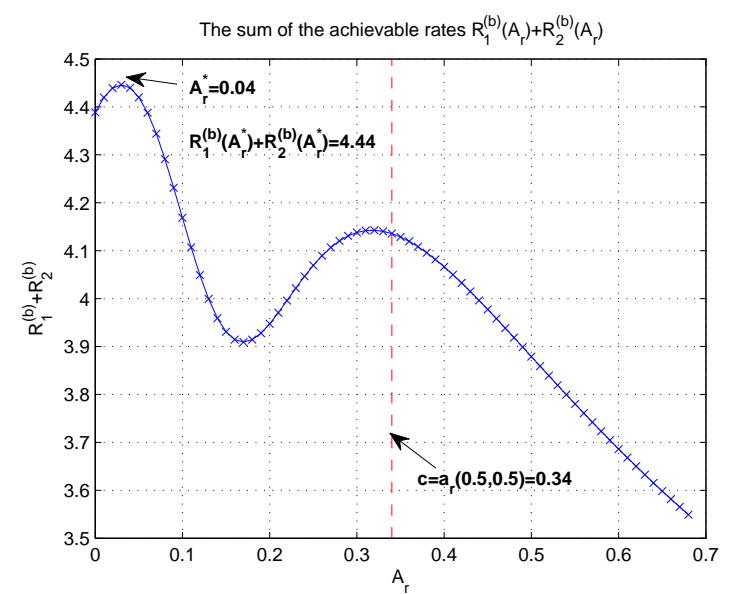

Fig. 4. Amplify-and-Forward, case of fixed amplification gain. The achievable rates $R_{1}^{(b)}+R_{2}^{(b)}$ as a function of the amplification gain $A r \in\left[0, \bar{a}_{r}\right]$. Saturating the relay power constraint is not optimal.

[4] O. Sahin and E. Erkip, "On Achievable Rates for Interference Relay Channel with Interference cancellation", Asilomar Conf. on Signals, Systems and Computers, pp. 805-809, Nov. 2007.

[5] O. Sahin and E. Erkip, "Achievable Rates for the Gaussian Interference Relay Channel", Globecom, pp. 1627-1631, 2007.

[6] W. Yu, G. Ginis and J. M. Cioffi, "Distributed multiuser power control for digital subscriber lines", IEEE J. of Sel. Areas in Comm., Vol. 20, No. 5, pp. 1105-1115, June 2002.

[7] J.-S. Pang, G. Scutari, F. Facchinei, C. Wang, "Distributed Power Allocation with Rate Constraints in Gaussian Parallel Interference Channels", IEEE Trans. on Inform. Theory, Vol. 54, No. 8, pp. 34713489, Aug. 2008.

[8] E. V. Belmega, B. Djeumou and S. Lasaulce, "What happens when cognitive terminals compete for a relay node?", IEEE International Conference on Accoustics, Speech and Signal Processing (ICASSP), Taipei, Taiwan, pp. 1-4, 19-24 April 2009.

[9] T. M. Cover and A. A. El Gamal, "Capacity theorems for the relay channel", IEEE Trans. Inform. Theory, IT-25(5): 572-584, 1979.

[10] J. F. Nash, "Equilibrium points in n-points games", Proc. of the Nat. Academy of Science, Vol. 36, no. 1, pp. 48-49, Jan. 1950.

[11] J. Rosen, "Existence and uniqueness of equilibrium points for concave n-person games", Econometrica, Vol. 33, pp. 520-534, 1965.

[12] A. Cournot, "Recherches sur les principes mathématiques de la la théorie des richesses, 1838, re-edited by Mac Millan in 1987.
[13] H. Moulin, "Dominance Solvability and Cournot Stability", Mathematical Social Sciences, Vol. 7, pp. 83-102, 1984.

[14] G. Kramer, M. Gastpar and P. Gupta, "Cooperative strategies and capacity theorems for relay networks", IEEE Trans. on Information Theory, Vol. 51, No. 9, pp. 3037-3063.

[15] S. Lasaulce, Y. Hayel, R. El Azouzi and M. Debbah, "Introducing hierarchy in energy games", IEEE Transactions on Wireless Communications, under revision, 2009.

[16] I. Abou-Faycal and M. Médard, "Optimal uncoded regeneration for binary antipodal signaling", IEEE Internaional Conference on Communications, Vol 2., pp.742-746, June 2004.

[17] K. S. Gomadam and S. A. Jafar, "On the capacity of memoryless relay networks", IEEE International Conference on Communication, June 2006.

[18] B. Djeumou, S. Lasaulce and A. G. Klein, "Practical Quantize-andForward Schemes for the Frequency Division Relay Channel", Eurasip Journal on Wireless Communications and Networking, Vol. 2007, Art. ID 20258, doi: 10.1155/2007/20258, Nov. 2007.

[19] M. A. Khojastepour, A. Sabharwal and B. Aazhang, "Lower bounds on the Capacity of Gaussian Relay Channel", http://hdl.handle.net/1911/20018, March 2004. 